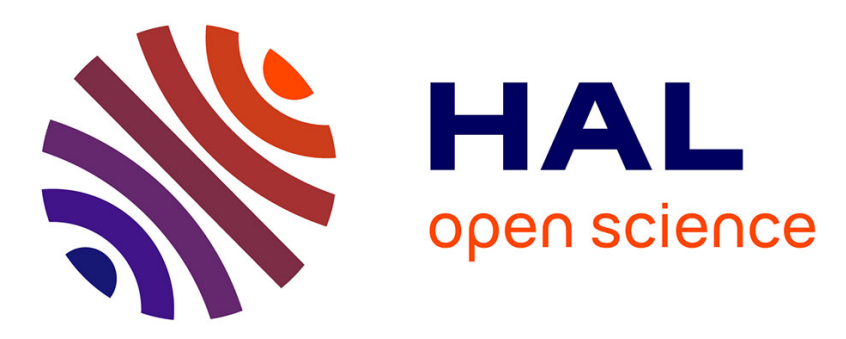

\title{
Stochastic feeding of fish larvae and their metabolic handling of starvation
}

Starrlight Augustine, Litvak K. Matt, Sebastiaan A.L.M. Kooijman

\section{To cite this version:}

Starrlight Augustine, Litvak K. Matt, Sebastiaan A.L.M. Kooijman. Stochastic feeding of fish larvae and their metabolic handling of starvation. Journal of Sea Research (JSR), 2011, 66, pp.411-418. 10.1016/j.seares.2011.07.006 . hal-00760060

\section{HAL Id: hal-00760060 https://hal.science/hal-00760060}

Submitted on 3 Dec 2012

HAL is a multi-disciplinary open access archive for the deposit and dissemination of scientific research documents, whether they are published or not. The documents may come from teaching and research institutions in France or abroad, or from public or private research centers.
L'archive ouverte pluridisciplinaire HAL, est destinée au dépôt et à la diffusion de documents scientifiques de niveau recherche, publiés ou non, émanant des établissements d'enseignement et de recherche français ou étrangers, des laboratoires publics ou privés. 


\title{
Stochastic feeding of fish larvae and their metabolic handling of starvation
}

December 3, 2012

\author{
S. Augustine*1, M. K. Litvak ${ }^{\dagger}$ and S.A.L.M. Kooijman ${ }^{\ddagger}$ \\ * Laboratory of Radioecology and Ecotoxicology, DEI/SECRE/LRE, Institute of Radioprotection and Nuclear \\ Safety (IRSN), Caradache, Building 186, BP3, 13115 St-Paul-lez-Durance Cedex, France e-mail: \\ starr-light.augusine@irsn.fr, Tel.: +33 442199413, Fax: +33 44219949. \\ $\dagger$ Department of Biology, Mount Allison University, 63B York St Sackville NB, E4L 1G7, Canada. e-mail: \\ mlitvak@mta.ca \\ $\ddagger$ Department of Theoretical Biology, Vrije Universiteit, de Boelelaan 1087, 1081 HV Amsterdam, the \\ Netherlands e-mail: bas@bio.vu.nl
}

This documents is the original paper before typesetting by Elsevier

\footnotetext{
${ }^{1}$ corresponding author
} 


\begin{abstract}
Developmental patterns of yolk-sac larvae are well captured by the standard DEB model: (i) when feeding is delayed post birth the size at which post-feeding growth begins is reduced but the rate of growth post-feeding is unaffected and (ii) maternal effects (initial energy in egg) show up as differences in condition at birth and maximum length of non fed individuals. We extended the standard DEB model in two ways to account for starvation. (I): if somatic maintenance can no longer be paid structure is also mobilized to cover the costs, but at an extra cost- conversion efficiency of structure to energy. Death occurs if structure reaches a fraction of the maximum at the onset of shrinking. (II): if maturity maintenance can no longer be paid then maturity level decays exponentially (rejuvenation). Hazard due to rejuvenation is proportional to the difference between maturity and the maximum maturity at the onset of rejuvenation.

We performed Monte Carlo simulation studies which treat feeding as a random process to evaluate the contribution of the metabolic handling of starvation to early teleost life history. The simulations suggest that food density strongly impacts growth, energy reserves, mineral fluxes, hazard and mortality from shrinking. Environmental factors can soon override maternal induced differences between individuals. Moreover in the low food density, simulated individuals from eggs of lower caloric content experience mortality from shrinking earlier than their counterparts issued from higher energy eggs. Empirically observed patterns of real data, i.e. high scatter in respiration in combination with low scatter in lengths, can be expected when the metabolism is treated as a deterministic system while behaviourally controlled input is stochastic. At low food densities where mortality from shrinking reaches $10 \%$ almost all individuals experience hazard due to rejuvenation. This hazard is difficult to access experimentally but represents moments of heightened susceptibility to pathogens and toxicants and could be ecologically significant.
\end{abstract}

Key words: DEB, starvation, stochastic feeding, rejuvenation, respiration, fish 


\section{List of Figures}

1 Modified from Kamler (2005). Egg size is measured in many different ways. Assumptions of auxiliary theory link these measurements to amounts of reserve and structure (egg size to initial amount of energy in reserve), which comes with

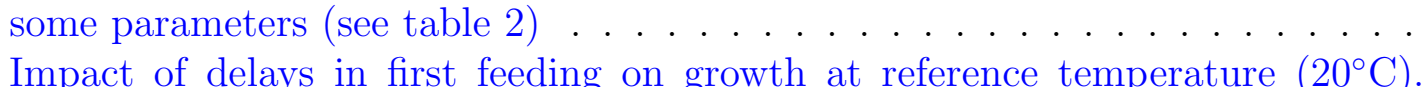
$s_{H}=2 ; \dot{k}_{J}^{\prime}=2.4 ; f=1$ (see table 2). (A) structural length (L) against age for $E_{0}=1.7$ (black lines) and $E_{0}=1.1$ (grey lines); arrowheads: birth; dashed lines: instantaneous death by shrinking. $E_{0}=1.7$ : feeding is never initiated (negative growth till death by shrinking) and feeding is delayed 0, 4 and 8 days after birth. $E_{0}=1.1$ : feeding feeding is never initiated and feeding is delayed 0 and 4 days after birth. Survival probability for $E_{0}=1.67(\mathrm{~B})$ and $E_{0}=1.1(\mathrm{C})$. Survival for delays in initial feeding of 0 days after birth $\left(^{*}\right), 4$ days after birth $(* *), 8$ days after birth $(* * *)$ and feeding is never initiated $(\dagger)$. Dotted line: all organisms have died from shrinking. . . . . . . . . . . . . . . . . length attained during development in the absence of feeding against yolk volume expressed in nanolitres (nl). Dashed line: model predictions of this maximum length for $1.1 \leq E_{0} \leq 2.5 \mathrm{~J}$. (B) observed length against age for eggs with all yolk volumes shown on left. Solid dark grey: model predictions supposing $\kappa_{G}=0.67$, solid light grey: model predictions supposing $\kappa_{G}=1$, dashed grey: death from shrinking in both cases, i.e. the length reached a fraction $\delta_{X}=0.75$ of the maximum at the onset of negative growth. . . . . . . . . . . . . . .

4 Model outputs computed at $20^{\circ} \mathrm{C} . s_{H}=2$ and $\dot{k}_{J}^{\prime}=0.024$ day $^{-1}$. First row: an example of scaled reserve density $(e)$, observed length $\left(L_{f}\right)$, wet mass, rejuvenation hazard $\left(\dot{h}_{J}\right)$ for an individual sampled randomly from the Monte Carlo simulation study. Second row: an example of fluxes of $\mathrm{CO}_{2}, \mathrm{H}_{2} \mathrm{O}, \mathrm{O}_{2}$ and $\mathrm{NH}_{3}$ against age since birth from the same individual sampled randomly from the Monte Carlo simulation study. Grey: stochastic mineral fluxes for that individual $\left(x=0.75, E_{0}=1.67 \mathrm{~J}, f\right.$ alternates between 0 and 1$)$ are compared to deterministic predictions (black smoth line) for $f=x /(1+x)$ and $f=0$ $\left(E_{0}=1.67 \mathrm{~J}\right)$. When $f=0$ the deterministic mineral fluxes go to 0 and the dotted line is when structure reaches a fraction $\delta_{X}=0.75$ of the maximum structure (instantaneous death is assumed at this point). Bottom row: distribution of each mineral flux at age 30 since birth for 5000 Monte Carlo trials $(x=0.75, E 0=1.67 \mathrm{~J}, f$ alternates between 0 and 1$)$. Please note that the $\mathrm{x}$ and $y$ axis of fluxes of $\mathrm{NH}_{3}$ differ. 
5 Output of 5000 Monte Carlo simulation studies at reference temperature $\left(20^{\circ} \mathrm{C}\right)$. $s_{H}=2$ and $\dot{k}_{J}^{\prime}=0.024$ day $^{-1}$. Open symbols: $x=0.75$, closed symbols $x=0.3$, circles: $E_{0}=1.8 \mathrm{~J}$, squares: $E_{0}=1.2 \mathrm{~J}$. (A) Distribution of lengths of live individuals after 30 days . (B) Distribution of wet mass of live individuals after 30 days. (C) Distribution of scaled reserve density $(e)$ of live individuals after 30 days. (D) Distribution of respiration $\left(\mathrm{nmol} \mathrm{O}_{2}\right.$ day $\left.^{-1}\right)$ of live individuals 30 days. (E) Age at death by shrinking for each individual. Black: $E_{0}^{1}$, grey: $E_{0}^{2}$. Note that no deaths occurred for $x=0.75$. (F) Percentage of live individuals who experience hazard due to rejuvenation during the simulation interval. Black: $E_{0}^{1}$, grey: $E_{0}^{2}$. No hazard is experienced for individuals in $E_{0}^{1}, x=0.75$. Percentage

of population in $E_{0}^{2}, x=0.75$ is barely perceptible on the graph. . . . . . . 16

\section{List of Tables}

1 State variables of the DEB model. . . . . . . . . . . . . . . . 8

2 Model parameters (affecting state variables at $20^{\circ} \mathrm{C}$ ) . . . . . . . . . . . 9 


\section{Introduction}

The ecology of teleost larvae has generated much interest in marine fisheries research. Survival through the early life history stages is thought to regulate recruitment and subsequent yearclass strength (Hjort, 1914; May, 1974; Cushing, 1975; Bailey and Houde, 1989, among others). Starvation (Hjort, 1914) and predation (Bailey and Houde, 1989) have been hypothesized as major factors controlling. Predation has been considered to be the primary agent of mortality during the yolk-sac stage (Blaxter and Fuiman, 1990; Paradis et al., 1996) while starvation may only become important after the transition to exogenous feeding (Leggett and Deblois, 1994) as there are potential interactions of larval size (Miller et al., 1988), developmental rate (Houde, 1987), environmental effects (Shepherd et al., 2000), predation and starvation. To date, with much of the emphasis focused on how growth and survival relate to environmental conditions, few studies consider how larval metabolism handles starvation.

The focus of our paper is (i) to formalise starvation rules which operate at the individual level and (ii) further investigate the importance of metabolism on starvation during teleost early life history stages. The study is conducted within the conceptual framework of Dynamic Energy Budget (DEB) theory (Sousa et al., 2008; Kooijman, 2010; Sousa et al., 2010): a well-tested theory on the uptake and use of substrate by all organisms. Parameters for the standard DEB model (see Kooijman, 2010, Chap.2) have been estimated for a number of animal species and can be found in the Add_My_Pet collection http://www.bio.vu.nl/thb/deb/deblab/add_my_ pet/ (Lika et al., 2011, this special issue). The standard DEB model is extended in this paper to deal with starvation in more detail. The question we ask is how does the metabolic handling of starvation contribute to growth, survival, dioxygen consumption, and carbon dioxide, water and ammonia production (hereafter referred to as mineral fluxes) during the early life history of the individual when resources are fluctuating. It is practical to conduct this study using a DEB model where the parameters of a teleost have already been estimated as well as with data which relate egg size to growth and survival. A full life cycle model for zebrafish, Danio rerio was previously developed (Augustine et al., 2011) and Jardine and Litvak (2003) have unique results where initial egg size was controlled by microinjection technique and lengths, yolk volumes and survival were observed for each individual. This makes zebrafish an ideal candidate for conducting theoretical studies on starvation.

Within the context of DEB theory the life cycle is divided in three stages: embryo (no assimilation), juvenile (assimilation) and adult (allocation to reproduction but no longer to maturation). Two important events bound the embryonic stage: (i) age zero where reserve is maximal and starts to reduce and structure and maturity are close to zero and start to increase and (ii) age at birth where external feeding is initiated and the embryo switches to a juvenile. Hatch precedes birth for many teleost species since the mouth is not yet formed and the individual is still reabsorbing the yolk sac inherited from the mother. Birth is initiated 
the moment the oesophagus opens and the digestive tract is open to the environment. Birth corresponds to a specific developmental milestone and so it makes sense to compare ages and lengths at this point in development between different individuals to understand maternal effects. Age and length at hatch is a more commonly found endpoint, but has the drawback of preceding birth and not occurring at strictly the same stage of development for all individuals (e.g. Kimmel et al., 1995, for zebrafish). Furthermore, age at hatch can be influenced by rearing protocol: sterilisation procedures such as bleaching can delay hatching by hardening the chorion (e.g. Zhang et al., 2009). Yolk consists of lipo-proteins, and during embryonic development it is rapidly converted into proteins, lipids and carbohydrates while hardly loosing energy or building blocks. These three products are stored in different places in the body, a process known as internalisation of yolk. These 'details' are not included in the standard model explicitly, and the yolk-sac is treated as a temporary organ with specialised functions, similar to adipose tissue of juveniles and adults in many taxa.

We first examine to what extent the DEB model is representative of impacts of maternal effects (initial egg caloric content) and delays in initial feeding on early juvenile development. Predictions are compared to actual observations of length against age of individuals born of eggs with initial yolk volumes ranging from 126 to $628 \mathrm{nl}$ (see Jardine and Litvak, 2003, for detailed protocol).

It has already been shown that stochastic food availability can explain the large variance in size of individuals in a same aquarium from a same brood due to amplification of difference by social interaction (Kooijman, 2009a). Large variation in larval zebrafish lengths (and high mortalities) were demonstrated to be a consequence of low food density, which was amplified by changes in food types, from small to larger food particles (Eaton and Farley, 1974). To emulate a random encounter rate between food items and juveniles we consider a natural stochastic version of the feeding module and perform Monte Carlo simulation studies to generate lengths, mass, reserve, mineral fluxes and survival for 5000 individuals. We perform the studies at low

and high food densities and with populations born of different initial egg energy content. The importance of including the metabolic handling of starvation when studying the ecology of juvenile teleosts is discussed.

\section{$2 \quad$ Model and methods}

\subsection{Formulation of starvation rules}

We use embryo parameter values for zebrafish provided in Augustine et al. (2011) while simplifying their model by neglecting deviations from isomorphy and effects of ageing. We also ignore any surface-area linked maintenance costs. As mentioned before, we focus on the early juvenile that follow the standard DEB model (Kooijman, 2010) and have two life stages (embryo 
and juvenile) using 3 state variables: energy in reserve $E(\mathrm{~J})$, structural length $L(\mathrm{~cm})$ and maturity $E_{H}$ (quantified in $\mathrm{J}$ ) (see table 1) and 7 parameters (see table 2). $L=V^{1 / 3}$ where $V$ $\left(\mathrm{cm}^{3}\right)$ is the structural biovolume.

The assimilation flux $\dot{p}_{A}\left(\mathrm{~J}_{\text {day }}{ }^{-1}\right)$ is

$$
\dot{p}_{A}=0 \quad \text { for embryos and } \quad \dot{p}_{A}=f\left\{\dot{p}_{A m}\right\} L^{2} \quad \text { for juveniles }
$$

with $\left\{\dot{p}_{A m}\right\}$ the maximum (surface-area) specific assimilation rate, and $f$ the scaled functional response, defined as the feeding rate on a particular food type as a fraction of the maximum possible one for an individual of that size. It can be specific for a particular diet. The standard model uses the Holling type II function response, $f=x /(1+x)$, with $x$ the scaled food density.

A fraction $\kappa$ of the flux $\dot{p}_{C}\left(\mathrm{~J} \mathrm{~d}^{-1}\right)$ that is mobilized from reserve is invested in somatic maintenance $\dot{p}_{M}=\left[\dot{p}_{M}\right] V$ and growth $\dot{p}_{G}=\kappa \dot{p}_{C}-\dot{p}_{M}$. A fraction $\kappa_{G}$ (the conversion efficiency) of the flux allocated to growth is fixed in new structure. $\kappa_{G}=\bar{\mu}_{V} d_{V}\left(\left[E_{G}\right] w_{V}\right)^{-1}$ (Lika et al., 2011 , this special issue), with $\left[E_{G}\right]$ the cost per unit of structure and $\bar{\mu}_{V}, d_{V}, w_{V}$ the chemical potential, density and molar weight of structure (see table 2).

A fraction $(1-\kappa) \dot{p}_{C}$ is invested in maturity maintenance $\dot{p}_{J}=\dot{k}_{J} E_{H}$ and maturation $\dot{p}_{R}=(1-\kappa) \dot{p}_{C}-\dot{p}_{J}$. Maturity does not have mass or energy itself, but is quantified as the cumulative energy investment in maturation. Maturity maintenance can be conceived as all processes responsible for maintaining the current state of maturity, i.e. immune, hormonal and cellular defence systems. An analogy would be that considerable energy is invested in learning and if the knowledge level is not maintained it is forgotten.

The mobilization flux is given by $\dot{p}_{C}=E(\dot{v} / L-\dot{r})$, with $\dot{v}\left(\mathrm{~cm} \mathrm{~d}^{-1}\right)$ the energy conductance and $\dot{r}\left(\mathrm{~d}^{-1}\right)$ the specific growth rate. Reserve dynamics are specified as follows: $\frac{d}{d t} E=\dot{p}_{A}-\dot{p}_{C}$.

Maintenance always has priority over investment in growth or maturation. Starvation occurs when energy mobilised no longer suffices to cover (I) the somatic maintenance cost, i.e. $\kappa \dot{p}_{C}<$ $\left[\dot{p}_{M}\right] V$, with shrinking as result and (II) the maturity maintenance costs, i.e. $(1-\kappa) \dot{p}_{C}<\dot{k}_{J} E_{H}$, with rejuvenation as result. It seems reasonable to assume that shrinking has a maximum and death occurs instantaneously when it exceeds a fixed fraction $\delta_{X}$. This construct comes with the need to introduce a new state variable $\max L$, i.e. the maximum length the individual once had. It seems likely that maturity maintenance is more optional than somatic maintenance in the sense that paying for the immune or the cellular defence systems might not be obligatory. Yet there is a penalty in the form of an increased hazard proportional to the fraction of maturity maintenance that is not paid (assuming implicitly that there is a constant need for defence). Also this construct comes with a need to introduce a new state variable $\max E_{H}$, i.e. the maximum maturity level the individual once had.

Both shrinking and rejuvenation have an extra parameter in the specification of their dynamics. On the assumption that the somatic maintenance costs expressed as energy flux is the same for growth and shrinking, the change in structural length is $\frac{d}{d t} L=L \dot{r} / 3$ with specific 
growth rate

$$
\dot{r}=\dot{k}_{M} g \frac{e / l-1}{e+g} \quad \text { if positive, else } \dot{r}=\dot{k}_{M} g \frac{e / l-1}{e+\kappa_{G} g}
$$

where $l=L / L_{m}$ is scaled length and $e=\frac{E \dot{v}}{L^{3}\left\{\dot{p}_{A m}\right\}}$ is scaled reserve density. The somatic maintenance rate coefficient $\dot{k}_{M}=\left[\dot{p}_{M}\right] /\left[E_{G}\right]$, the ultimate structural length $L_{m}=\kappa \frac{\left\{\dot{p}_{A m}\right\}}{\left[\dot{p}_{M}\right]}$ and the energy investment ratio $g=\frac{\left[E_{G}\right] \dot{v}}{\kappa\left\{\dot{p}_{A m}\right\}}$ are compound parameters of the standard DEB model. Somatic maintenance might have a 'building block' aspect that might cause growth efficiency $\kappa_{G}$ to deviate from the value mentioned above.

Following the previous analogy we can imagine that forgetting follows a first order process with rate parameter $\dot{k}_{J}^{\prime}$, say:

$$
\frac{d E_{H}}{d t}=(1-\kappa) \dot{p}_{C}-\dot{k}_{J} E_{H} \quad \text { if positive, else } \frac{d E_{H}}{d t}=-\dot{k}_{J}^{\prime}\left(E_{H}-\frac{(1-\kappa) \dot{p}_{C}}{\dot{k}_{J}}\right)
$$

Hazard due to rejuvenation $\dot{h}_{J}$ is considered proportional to the difference between actual maturity level and maximum maturity level at the onset of rejuvenation max $E_{H}$ :

$$
\dot{h}_{J}=\dot{k}_{M} \frac{\max E_{H}-E_{H}}{E_{H}^{h}}
$$

and

$$
E_{H}^{h}=\frac{(1-\kappa)\left[E_{G}\right] L_{m}^{3}}{\kappa s_{H}}
$$

where $s_{H}$ is the rejuvenation stress coefficient. The result is that $\dot{h}_{J}$ contributes to survival as:

$$
\frac{d S}{d t}=-S\left(\dot{h}_{J}+\dot{h}_{a}+\dot{h}_{\mathrm{acc}}+\dot{h}_{\mathrm{sh}}\right)
$$

In this study we consider that hazard from ageing $\dot{h}_{a}$ and from accidents $\dot{h}_{\text {acc }}$ is negligible. We consider that hazard from shrinking of structure $\dot{h}_{\text {sh }}$ is infinite with instantaneous death as a result when the shrinking threshold is passed.

The starvation module has, in summary, two new state variables, $\max L$ and $\max E_{H}$, and four new parameters $\kappa_{G}, \delta_{X}, \dot{k}_{J}^{\prime}$ and $s_{H}$. Growth efficiency also occurs under non-starvation conditions, but there it does not play a role in the dynamics of the state variables.

Embryo and juvenile mineral fluxes are specified in the Appendix. For a full discussion we refer to Kooijman (2010, Chap.4). 
Table 1: State variables of the DEB model.

\begin{tabular}{|c|c|c|}
\hline Variable & Unit & Name \\
\hline \multicolumn{3}{|c|}{ Standard DEB model } \\
\hline$E$ & $\mathrm{~J}$ & reserve \\
\hline$L$ & $\mathrm{~cm}$ & structural length \\
\hline$E_{H}$ & $\mathrm{~J}$ & cumulated energy invested in maturity \\
\hline \multicolumn{3}{|c|}{ Forcing variable } \\
\hline$x$ & - & scaled food density \\
\hline \multicolumn{3}{|c|}{ Additional state variables } \\
\hline $\begin{array}{l}\max L \\
\max E_{H}\end{array}$ & $\begin{array}{l}\mathrm{cm} \\
\mathrm{J}\end{array}$ & $\begin{array}{l}\text { maximum structural length before shrinking } \\
\text { maximum } E_{H} \text { before rejuvenation }\end{array}$ \\
\hline
\end{tabular}

\subsection{Linking state variables to measurements}

Structural length $L$ is taken proportional to some well-chosen physical (observed) length $L_{f}$ : $L=\delta_{M} L_{f}$, with $\delta_{M}$ the (constant) shape correction factor. Augustine et al. (2011) show that the shape of early juvenile zebrafish changes during the first month of development. This is most likely the case for many teleost species who undergo metamorphosis after early juvenile development. We exclude this detail from our study by considering that $\delta_{M}$ is constant.

Freshly spawned eggs are taken to be composed uniquely of reserve inherited from the mother. Maternal effects are expressed as differences in the initial energy content of the egg $E_{0}$ $(\mathrm{J})$. We relate observed egg volume to initial energy content as follows:

$$
V_{f}^{Y}=\frac{E_{0}}{\delta_{Y}^{3}} \frac{w_{E}}{\bar{\mu}_{E} d_{E}}
$$

$\delta_{Y}$ is an empirical shape correction factor. $w_{E}, d_{E}$ and $\bar{\mu}_{E}$ are the molar weight, density and chemical potential of reserve (table 2). We assume an Arrhenius relationship between metabolic rates and temperature (Eqn. 1.2 Kooijman, 2010). Computations are made at reference temperature of $20^{\circ} \mathrm{C}$. Predictions which are compared to observations by Jardine and Litvak (2003) are temperature corrected to $28^{\circ} \mathrm{C}$.

Dry biomass $W_{d}$ has contributions from both reserve and structure and is expressed as:

$$
W_{d}=d_{V} L^{3}+\frac{w_{E}}{\bar{\mu}_{E}} E
$$

with $d_{V}$ the density of structure (table 2). We make the simplified assumption that wet biomass $W_{w}$ is equal to $6 W_{d}$ on the basis of observations by Craig and Fletcher (1984); Bagatto et al. (2001). 
Table 2: Model parameters (affecting state variables at $20^{\circ} \mathrm{C}$ )

\begin{tabular}{|c|c|c|c|}
\hline Parameter & Value & Unit & Name \\
\hline \multicolumn{4}{|c|}{ DEB model parameters (values taken from Augustine et al., 2011) } \\
\hline$\left\{\dot{p}_{A m}\right\}$ & 246.3 & $\mathrm{~J}$ day $^{-1} \mathrm{~cm}^{-2}$ & maximum surface-area assimilation \\
\hline & 0.0287 & $\mathrm{~cm} \mathrm{day}^{-1}$ & energy conductance \\
\hline$\left[\dot{p}_{M}\right]$ & 500.9 & $\mathrm{~J}_{\text {day }}{ }^{-1} \mathrm{~cm}^{-3}$ & volume-linked somatic maintenance costs \\
\hline$\left[E_{G}\right]$ & 4652 & $\mathrm{~J} \mathrm{~cm}^{-3}$ & cost of a unit of structure \\
\hline$\dot{k}_{J}$ & 0.0166 & day -1 & maturity maintenance rate \\
\hline$\kappa$ & 0.44 & - & allocation fraction to soma \\
\hline$E_{H}^{b}$ & 0.54 & $\mathrm{~J}$ & cumulated energy invested in maturity at birth \\
\hline \multicolumn{4}{|c|}{ Temperature correction module (values taken from Augustine et al., 2011) } \\
\hline$T_{A}$ & 3000 & $\mathrm{~K}$ & Arrhenius temperature \\
\hline \multicolumn{4}{|c|}{ Auxiliary parameters (values taken from Lika et al., 2011, this special issue) } \\
\hline$w_{E}$ & 23.9 & $\mathrm{~g} \mathrm{C}^{-\mathrm{mol}^{-1}}$ & molar weight of reserve \\
\hline$w_{V}$ & 23.9 & $\mathrm{~g} \mathrm{C}^{-\mathrm{mol}^{-1}}$ & molar weight of structure \\
\hline$d_{E}$ & 0.15 & $\mathrm{~g} \mathrm{~cm}^{-3}$ & density of reserve \\
\hline$d_{V}$ & 0.15 & $\mathrm{~g} \mathrm{~cm}^{-3}$ & density of structure \\
\hline $\bar{\mu}_{E}$ & 555 & $\mathrm{~kJ} \mathrm{C}-\mathrm{mol}^{-1}$ & chemical potential of reserve \\
\hline $\bar{\mu}_{V}$ & 500 & $\mathrm{~kJ} \mathrm{C}-\mathrm{mol}^{-1}$ & chemical potential of structure \\
\hline \multicolumn{4}{|c|}{ Parameters linked to starvation (this study) } \\
\hline$\dot{k}_{J}^{\prime}$ & depends on study & day $^{-1}$ & specific maturity decay \\
\hline$s_{H}$ & depends on study & - & rejuvenation stress coefficient \\
\hline$\delta_{X}$ & 0.75 & - & maximum shrinking fraction \\
\hline \multicolumn{4}{|c|}{ Parameters linked to respiration(this study) } \\
\hline$y_{X E}$ & 1.25 & $\mathrm{C}-\mathrm{mol} \mathrm{X} / \mathrm{C}-\mathrm{mol} \mathrm{E}$ & yield of food on reserve \\
\hline$y_{P X}$ & 0.1 & C-mol P/ C-mol X & yield of faeces on food \\
\hline \multicolumn{4}{|c|}{ Shape coefficients used for comparing model output to data from Jardine and Litvak (2003) } \\
\hline$\delta_{Y}$ & 1.2521 & - & yolk shape coefficient \\
\hline$\delta_{M}$ & 0.1245 & - & shape coefficient \\
\hline \multicolumn{4}{|c|}{ Stochastic feeding module } \\
\hline$M_{X}$ & 0.06 & $\mu \mathrm{mol}$ & Mass of food particle \\
\hline
\end{tabular}




\subsection{Stochastic feeding module}

Suppose that food density, in terms of particles per volume, is constant, such as in a polyculture (e.g. Best et al., 2010). We simplify the system by considering all food items to be of constant chemical composition with mass $M_{X}(\mu \mathrm{mol})$. Each fish is either searching for food (i.e. $f=0$ ) or handling food (i.e. $f=1$ ). Time interval spent handling food $t_{h}$ is inversely proportional to squared length (of the individual), so larger individuals take less time to handle a food item. $\left\{\dot{J}_{X A m}\right\}$ is the surface-area specific maximum intake rate (in C-mol food per day per surface area) and relates to surface-area maximum assimilation rate $\left\{\dot{p}_{A m}\right\}$ as: $\left\{\dot{J}_{X A m}\right\}=$ $\left\{\dot{p}_{A m}\right\} y_{X E} / \bar{\mu}_{E} \cdot y_{X E}$ is the yield of food on reserve (see table 2).

$$
t_{h}=\frac{M_{X}}{\left\{\dot{J}_{X A m}\right\} L^{2}}
$$

Food searching intervals $t_{s}$ are considered as an exponentially distributed random variable with mean $t_{h} / x$. Mean feeding rate is defined as $\dot{h}_{X}=\left(t_{s}+t_{h}\right)^{-1}=f / t_{h}$. This natural introduction of stochasticity does not come with any new parameters.

Two populations are created from two pools of initial energy contents: $E_{0}^{1}=1.18 \mathrm{~J}$ and $E_{0}^{2}=1.68 \mathrm{~J}$. Populations $E_{0}^{1}$ and $E_{0}^{2}$ both develop in two different environments: $x_{1}=0.3$ and $x_{2}=0.75$. This makes 4 experimental conditions: (i) $E_{0}^{1}, x_{1}$, (ii) $E_{0}^{1}, x_{2}$, (iii) $E_{0}^{2}, x_{1}$, and (iv) $E_{0}^{2}, x_{2}$. We run 5000 Monte Carlo simulation for each condition and represent results as the distributions of lengths, mass, scaled reserve density $e$, and respiration at 30 days since birth. In addition, we look at the distribution of age at death from shrinking for the entire study and the number of live individuals at 30 days since birth who have experienced rejuvenation hazard. Stochastic simulation routines (traject.m and traject_m.m) are part of the DEBtool software (Kooijman et al., 2008) and are freely downloadable at http://www.bio.vu.nl/thb/ research/.

\section{Results and Discussion}

\subsection{Constant food}

Model simulations show that when initial feeding is delayed post birth, size at which postfeeding growth begins is reduced but the rate of growth post-feeding is unaffected (figure $2 \mathrm{~A}$ ). This is in line with general observations by Guillaume et al. (1999). Individuals from the lower $E_{0}$ die from shrinking if feeding is delayed beyond 4 days. The initial caloric content of the egg influences post-birth lifespan when feeding is never initiated which is again in agreement with general patterns noted by Kamler (2005). Rejuvenation hazard contributes to reducing survival 


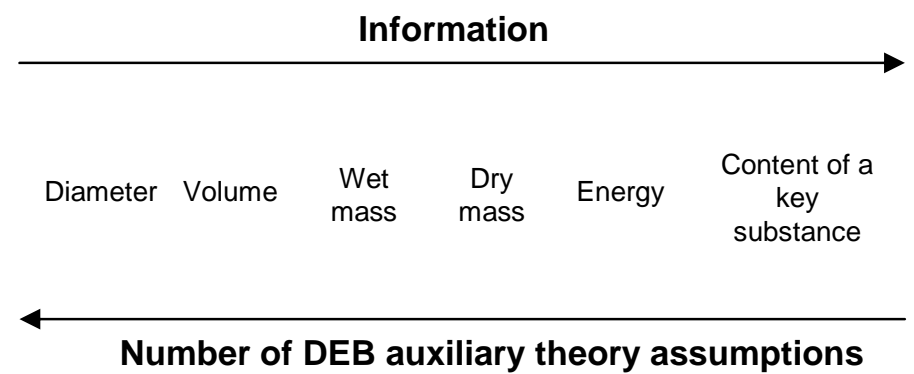

Figure 1: Modified from Kamler (2005). Egg size is measured in many different ways. Assumptions of auxiliary theory link these measurements to amounts of reserve and structure (egg size to initial amount of energy in reserve), which comes with some parameters (see table 2, auxiliary parameters).

probability (figure $2 \mathrm{~B}$ and $\mathrm{C}$ ). The time an individual can handle delays in initial feeding before rejuvenation hazard starts contributing to survival relates to initial energy in the egg.

The model predicts that maternal effects (initial energy in egg) influence condition at birth in terms of amount of reserve per unit structure and maximum length of non fed individuals. Birth does not occur if $E_{0}<1.1 \mathrm{~J}$ because the cumulated energy invested in maturity never attains the threshold value $E_{H}^{b}$ (see table 2). Hence eggs are considered non viable when $E_{0}<1.1 \mathrm{~J}$. It would be interesting to verify experimentally the realism of this lower boundary value of $E_{0}$ for which an egg is viable. Should such a lower boundary exist then maternal effects can also contribute to embryonic survival.

The maternal effect rule (Kooijman, 2009b), implemented by default into the standard DEB model, stipulates that the reserve density of the mother equals the reserve density of her offspring at birth. $E_{0}=1.67 \mathrm{~J}$ for mothers at $f=1$ (for this parameter combination). Between the narrow caloric range of $E_{0}=1.1$ to $1.67 \mathrm{~J}$, the maximum structure for a non fed juvenile increases by $10 \%$, and the maximum maturity attained increases by $40 \%$. This explains the increased hazard experienced by lower $E_{0}$ individuals during starvation (figure $2 \mathrm{C}$ ). Caloric values of egg dry mass for teleost species are generally very conserved ranging from $20-30$ $\mathrm{J} \mathrm{mg}^{-1}$ (Kamler, 2005). If we consider the dry mass of an egg as $W_{0}=E_{0} w_{E} / \bar{\mu}_{E}$, then the caloric value of $E_{0}=1.6 \mathrm{~J}$ is $22.9 \mathrm{~J} \mathrm{mg}^{-1}$ which falls within the boundaries suggested by Kamler (2005).

We do not propose a maximum egg size in this study. Links between egg size and the mother's condition might be more complex and depending on the applications deviations from 

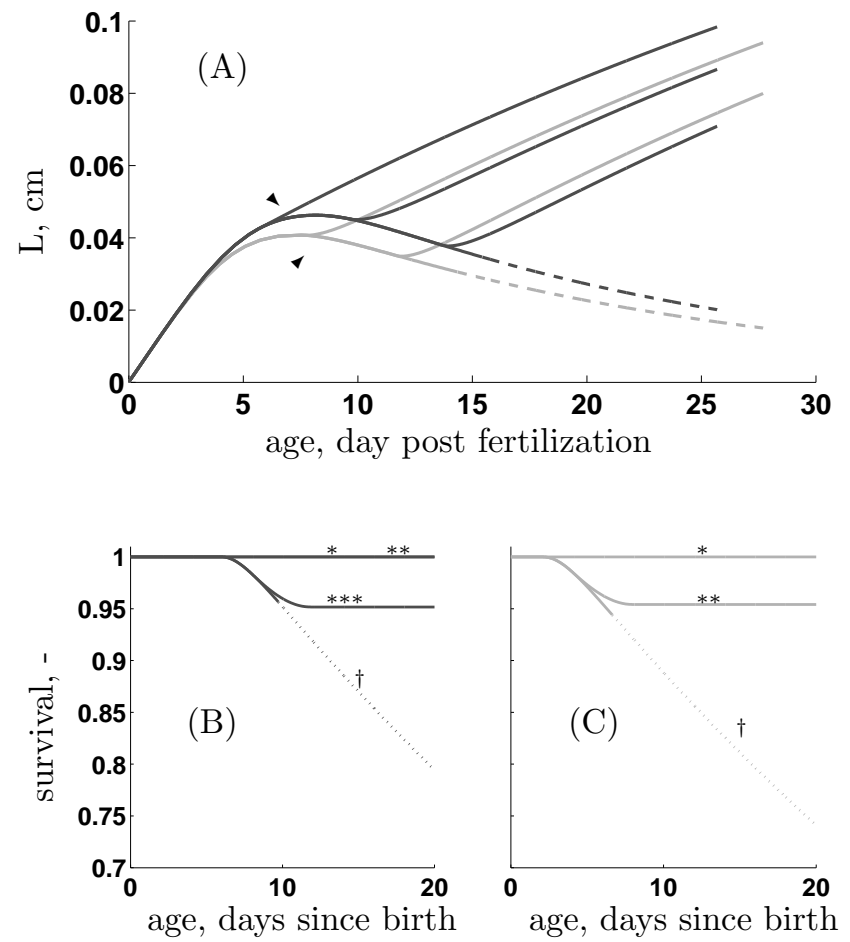

Figure 2: Impact of delays in first feeding on growth at reference temperature $\left(20^{\circ} \mathrm{C}\right) . s_{H}=2 ; \dot{k}_{J}^{\prime}=2.4 ; f=1$ (see table 2). (A) structural length (L) against age for $E_{0}=1.7$ (black lines) and $E_{0}=1.1$ (grey lines); arrowheads: birth; dashed lines: instantaneous death by shrinking. $E_{0}=1.7$ : feeding is never initiated (negative growth till death by shrinking) and feeding is delayed 0,4 and 8 days after birth. $E_{0}=1.1$ : feeding feeding is never initiated and feeding is delayed 0 and 4 days after birth. Survival probability for $E_{0}=1.67(\mathrm{~B})$ and $E_{0}=1.1(\mathrm{C})$. Survival for delays in initial feeding of 0 days after birth $\left(^{*}\right), 4$ days after birth $(* *), 8$ days after birth $(* * *)$ and feeding is never initiated $(\dagger)$. Dotted line: all organisms have died from shrinking.

the maternal effect rule should be considered. The maximum surface-area specific assimilation rate $\left\{\dot{p}_{A m}\right\}$ is sensitive to the type of food. Consequently, maximum assimilation rate which corresponds to $f=1$ for one type of food might correspond to $f<1$ for another. Since the maternal effect rule implies that the $e$ of the embryo at birth equals the value of $f$ of the mother at spawning and that diets differ between individuals, using $E_{0}>1.67 \mathrm{~J}$ as starting values to calculate life history traits is coherent with model assumptions.

In figure $3 \mathrm{~A}$ we computed lengths of non fed juvenile zebrafish for $1.1 \leq E_{0} \leq 2.5 \mathrm{~J}$ as suggested by the large range of observed yolk volumes $V_{f}^{Y}$. Predicted maximum length as a function of $V_{f}^{Y}$ is compared with the actual observations from Jardine and Litvak (2003). Maximum size attained seems to saturate for initial yolk volumes above $314 \mathrm{nl}$. This points to an uneasy relationship between yolk volume and water content. Large eggs tend to have higher caloric value than small eggs, but this is not always the case (Kamler, 2005). For Sardina pilchardus, Riveiro et al. (2000) found that that time to yolk absorption was not a function of egg size but a function of egg biochemical composition (protein content) which illustrates the point we make in figure 1 . We converted $E_{0}$ to $V_{f}^{Y}$ with the implicit assumption that the fraction of water per volume is constant. There is no solid evidence that this is the case. Still, the data are overall supportive of a positive relationship between $E_{0}$ and maximum length of a non fed individual. 
We further compare predicted lengths of individuals issued from eggs with energy content $1.1 \leq E_{0} \leq 2.5 \mathrm{~J}$ to observed lengths against age for each $V_{f}^{Y}$ (figure 3B). We show two cases: (i) paying maintenance from structure increases somatic maintenance costs by a factor $\kappa_{G}^{-1}$ and (ii) there is no extra cost involved $\left(\kappa_{G}=1\right)$ which is not thermodynamically coherent. In both cases predicted negative growth rate at the onset of maximum structure is overestimated. The discrepancy might be explained by parameters values. On the other hand, does an organism shrink isomorphically? Yin and Blaxter (1986) found that it was the ratios of morphometric measurements which were sensitive to the state of starvation for larval Gadus morhua L. and Platichthys flesus L.. A length measurement following the spinal cord, as is the case here, might be relatively insensitive to shrinking. Nonetheless, the model reflects the observed relationships between $V_{f}^{Y}$ and maximum lengths while lengths at starvation are underestimated.

The predicted effects of initial energy in egg on growth and metabolic handling of starvation lends support to current views where maternal effects contribute to resistance to starvation. Yet it remains difficult to assess the importance of this maternal effect in real life situations. We tackle the question theoretically using Monte Carlo simulations with stochastic food input in the following section.
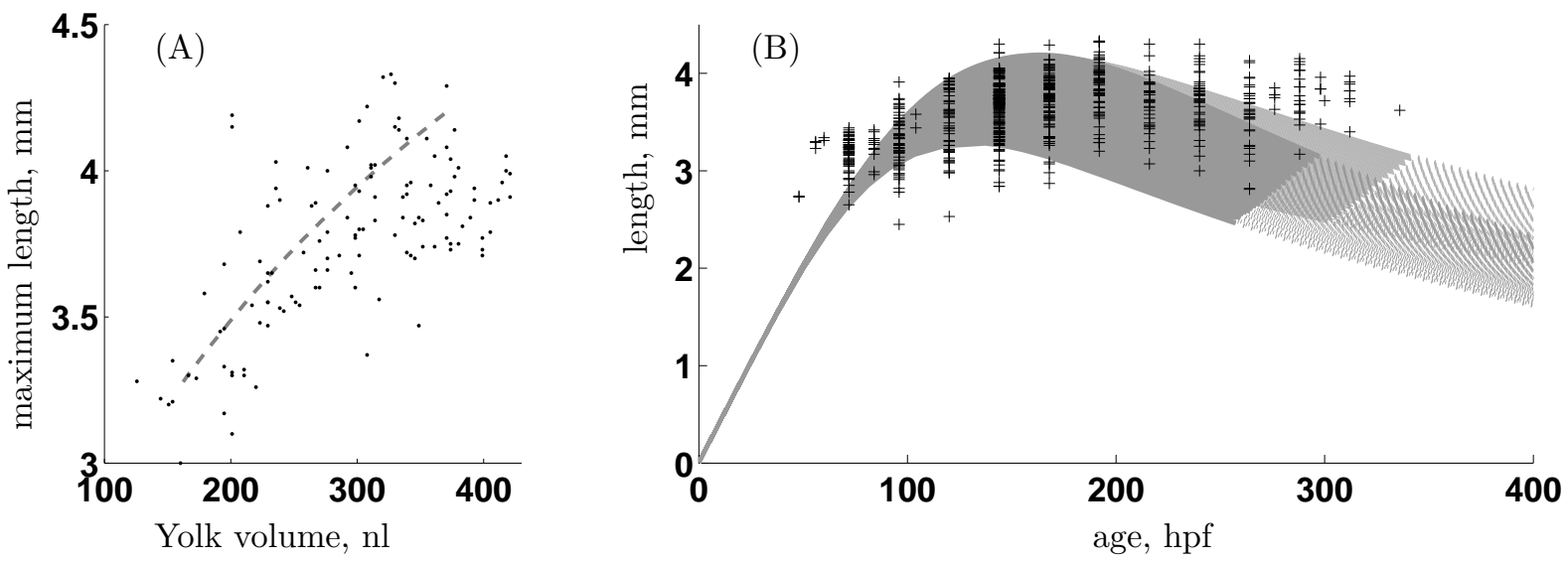

Figure 3: Points: data from Jardine and Litvak $(2003)\left(28^{\circ} \mathrm{C}\right)$. (A) maximum observed length attained during development in the absence of feeding against yolk volume expressed in nanolitres (nl). Dashed line: model predictions of this maximum length for $1.1 \leq E_{0} \leq 2.5 \mathrm{~J}$. (B) observed length against age (hours post fertilization hpf) for eggs with all yolk volumes shown on left. Solid dark grey: model predictions supposing $\kappa_{G}=0.67$, solid light grey: model predictions supposing $\kappa_{G}=1$, dashed grey: death from shrinking in both cases, i.e. the length reached a fraction $\delta_{X}=0.75$ of the maximum at the onset of negative growth. 


\subsection{Stochastic searching}

All stochasticity that we propose is in food searching, not in food handling. This is natural because we assume a fixed particle size for simplicity's reasons, consistent with the idea that the standard DEB model pushes simplicity into the extreme. In many natural situations, food particles will show a particle-size distribution, which introduces stochasticity in handling as well, but also comes with new parameters and the results become dependent on the particle size distribution, which itself will be affected by many factors.

The values of $e, L_{f}, W w, \dot{h}_{J}$ and mineral fluxes against age since birth of an individual experiencing a stochastic encounter with food particles in environment $E_{0}^{1}, x_{1}$ is compared to deterministic predictions for an individual at $f=x_{1} /\left(1+x_{1}\right)$ (expected mean) and a starved individual $(f=0)$. See figure 4 , first and second row.

Stochastic mineral fluxes show rapid small scale variation since $f$ is fluctuating continuously between 0 and 1 . The distribution of daily mineral production $\left(\mathrm{CO}_{2}, \mathrm{H}_{2} \mathrm{O}, \mathrm{NH}_{3}\right.$ figure $4 \mathrm{I}$, J and $\mathrm{L}$ ) or consumption $\left(\mathrm{O}_{2}\right.$ figure $\left.4 \mathrm{~K}\right)$ of 5000 individuals at the end of the simulation time shows considerable deviation from the mean. Future research however could include digestion (see e.g. Kooijman, 2010, Chap.7) which would make assimilation a smoother process at small time scales. Differences in prior food history will still accumulate however and contribute to inter-individual differences in lengths, mass, reserve and mineral production and consumption. The mineral flux curves all show the same morphology, but this is a result of choice of parameter values and not a model property. The mineral fluxes are closely tied to energetics and go to zero when the individual is starved. No individuals in the $x=0.75, E_{0}^{1}$ environment experience rejuvenation hazard. Figure $4 \mathrm{D}$ shows rejuvenation hazard when $f=0$ and the organism is shrinking.

The Monte Carlo simulation studies reveal the predicted scatter in lengths, weights, survival and respiration at different time points when food availability fluctuates. Monte Carlo results of simulations for each of the 500 individuals at day 30 since birth are presented in figure 5 . Interestingly the largest scatter is observed in the predicted mass and respiration data and the lowest in the predicted length data: the coefficient of variation $(\mathrm{CV})$ of mass and $\mathrm{O}_{2}$ consumption is almost four time times the $\mathrm{CV}$ of length in all cases. In the $x_{2}, E_{0}^{1}$ environment for example the $\mathrm{CV}$ is $9 \%$ for $L_{f}, 34 \%$ for $W w$ and $38 \%$ for $\mathrm{O}_{2}$ consumption. Monte Carlo simulations of DEB models with stochastic food input seem to inherently capture patterns of scatter observed in real data sets where high scatter in measured mass or respiration combines with much lower scatter in observed lengths.

The CV increases twofold between the high and the low food density environments for all predicted observations regardless of the initial energy content of the egg. For example, the CV of the $L_{f}$ distribution for $x_{1}, E_{0}^{1}$ is $4 \%$ wheareas it is $9 \%$ for $x_{2}, E_{0}^{1}$ (figure $5 \mathrm{~A}$ ). This links up beautifully with the results of Eaton and Farley (1974) where lengths of individuals sampled after 16 days of development in containers with 2 free-swimming prey per $\mathrm{mL}$ had a $\mathrm{CV}$ of 

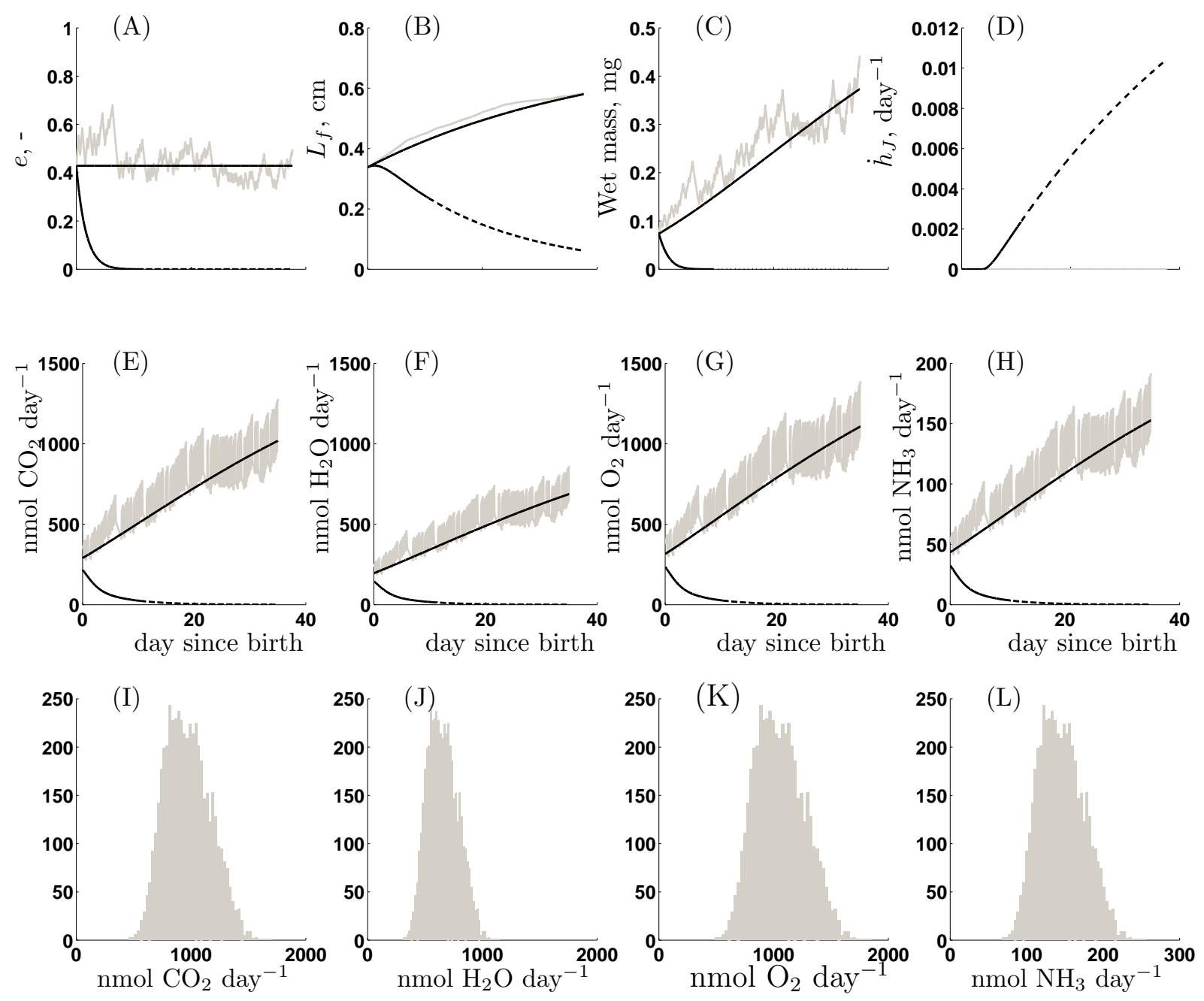

Figure 4: Model outputs computed at $20^{\circ} \mathrm{C} . s_{H}=2$ and $\dot{k}_{J}^{\prime}=0.024$ day $^{-1}$. First row: an example of scaled reserve density $(e)$, observed length $\left(L_{f}\right)$, wet mass, rejuvenation hazard $\left(\dot{h}_{J}\right)$ for an individual sampled randomly from the Monte Carlo simulation study. Second row: an example of fluxes of $\mathrm{CO}_{2}$, $\mathrm{H}_{2} \mathrm{O}, \mathrm{O}_{2}$ and $\mathrm{NH}_{3}$ against age since birth from the same individual sampled randomly from the Monte Carlo simulation study. Black: stochastic mineral fluxes for that individual $\left(x=0.75, E_{0}=1.67 \mathrm{~J}, f\right.$ alternates between 0 and 1 ) are compared to deterministic predictions (smooth lines) for $f=x /(1+x)$ and $f=0\left(E_{0}=1.67 \mathrm{~J}\right)$. When $f=0$ the deterministic mineral fluxes go to 0 and the dotted line is when structure reaches a fraction $\delta_{X}=0.75$ of the maximum structure (instantaneous death is assumed at this point). Bottom row: distribution of each mineral flux at age 30 since birth for 5000 Monte Carlo trials $(x=0.75, E 0=1.67 \mathrm{~J}, f$ alternates between 0 and 1$)$. Please note that the $\mathrm{x}$ and $y$ axis of fluxes of $\mathrm{NH}_{3}$ differ. 

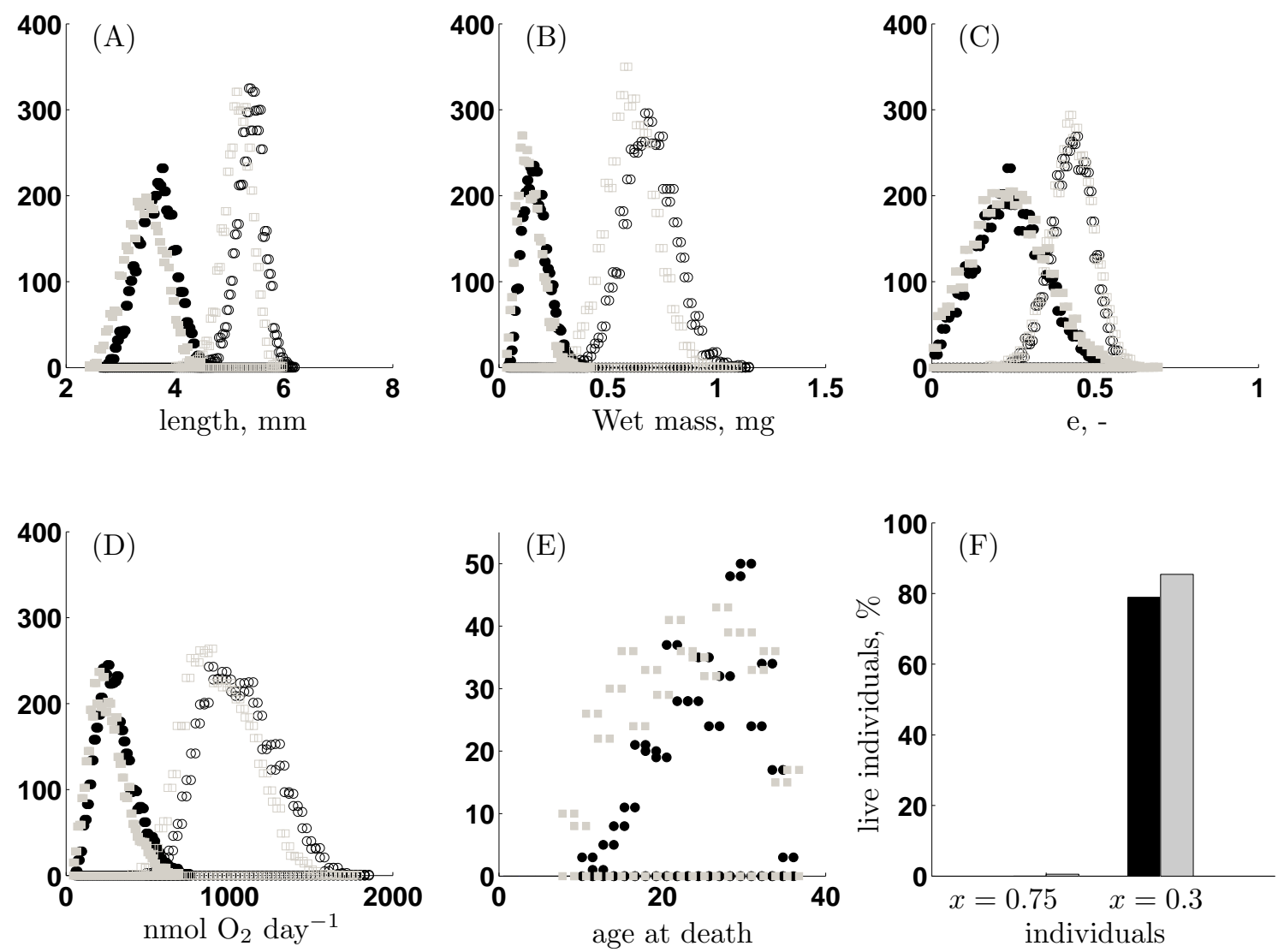

Figure 5: Output of 5000 Monte Carlo simulation studies at reference temperature $\left(20^{\circ} \mathrm{C}\right) . s_{H}=2$ and $\dot{k}_{J}^{\prime}=0.024$ day $^{-1}$. Open symbols: $x=0.75$, closed symbols $x=0.3$, circles: $E_{0}=1.8 \mathrm{~J}$, squares: $E_{0}=1.2 \mathrm{~J}$. (A) Distribution of lengths of live individuals after 30 days . (B) Distribution of wet mass of live individuals after 30 days. (C) Distribution of scaled reserve density $(e)$ of live individuals after 30 days. (D) Distribution of respiration $\left(\mathrm{nmol} \mathrm{O}_{2}\right.$ day $^{-1}$ ) for live individuals at day 30. (E) Age at death by shrinking for each individual. Black: $E_{0}^{1}$, grey: $E_{0}^{2}$. Note that no deaths occurred for $x=0.75$. (F) Percentage of live individuals who experience hazard due to rejuvenation during the simulation interval. Black: $E_{0}^{1}$, grey: $E_{0}^{2}$. No hazard is experienced for individuals in $E_{0}^{1}, x=0.75$. Percentage of population in $E_{0}^{2}, x=0.75$ is barely perceptible on the graph. 
$16.8 \%$ and lengths of individuals sampled after 15 days of development in containers holding 200-300 free-swimming prey per $\mathrm{mL}$ had a CV of $3.8 \%$.

Populations $E_{0}^{1}$ and $E_{0}^{2}$ evolve in a very similar manner in terms of lengths, weights, respiration and survival at both food densities $(x=0.3$ and $x=0.75)$. Thus the present simulations hint at a negligible contribution of maternal effects to measurable endpoints after a month of development regardless of the food density. Maternal effects can be diluted by the individuals (chance) encounter rate with food items.

The most sensitive difference between $E_{0}^{1}$ and $E_{0}^{2}$ at $x=0.3$ is in the distribution of age at death (figure $5 \mathrm{E}$ ). Population $E_{0}^{2}$ shows higher mortalities from age 8 to 20 since birth than the population $E_{0}^{1}$. After 20 days, age at death distributions are quasi identical between both populations. At $x=0.3$ about $10 \%$ of both populations die from structure shrinking. At $x=0.2$ about $50 \%$ of both populations die from shrinking (not shown).

Almost all individuals in both populations in the low food density environment $(x=0.3)$ experience hazard due to rejuvenation. This is shown in figure $5 \mathrm{~F}$ where $79 \%$ of live individuals experienced hazard in $E_{0}^{1}, x=0.3$ and $85 \%$ of live individuals experienced hazard in $E_{0}^{2}$, $x=0.3$. Rejuvenation hazard hardly occurred for individuals in $x_{1}, E_{0}^{1}$ and $x_{2}, E_{0}^{1}$. This hazard $\left(\dot{h}_{J}\right)$ can be translated biologically as moments of increased susceptibility to pathogens and stressors and contributes to survival probability. It is difficult to evaluate $\dot{h}_{J}$ experimentally, but the present simulations suggest that it could be a relevant factor regulating survival should additional environmental stressors and small periods of starvation occur simultaneously.

\section{Concluding remarks}

There exists strong empirical support for maternal effects as a general pattern but our simulations show how environmental conditions soon override such effects. Inter-individual differences in prior food history accumulate and can be responsible for the observed scatter in the data.

The problem of inherent variability in biological data must be addressed in all fields of biology. The present study shows that empirically observed patterns of real data, i.e. high scatter in respiration in combination with low scatter in lengths, can be expected when the metabolism is treated as a deterministic system while behaviourally controlled input is stochastic. This is consistent with general patterns in observations.

Monte Carlo simulation studies are powerful tools to understand how stochasticity of food searching translates into variability in measured endpoints such as respiration, growth or reproductive output. This insight can be useful for designing experiments: e.g. feeding protocols, types and frequencies of measurements and sampling.

The concept of rejuvenation and rejuvenation hazard follows directly from the structure of the DEB model but further theoretical work needs to be done to understand how to estimate it from data. The concept links up neatly with empirical support that starved organism show 
a heightened susceptibility to disease and environmental stressors such as toxicants (Rougier et al., 1996).

The extension we propose of the standard DEB model specifying shrinking and rejuvenation is not species-specific and applies to the entire life-cycle. Further dedicated research will test to what degree these rules are inherent to the functioning of all organisms and further our understanding of which adaptations are species specific and/ or specific to a particular life stage of the organism.

\section{Acknowledgements}

This work is part of the ENVIRHOM research program supported by the Institute for Radioprotection and Nuclear Safety and the Provence Alpes Côte d'Azur region. Béatrice Gagnaire and Christelle Adam-Guillermin are gratefully acknowledged for the stimulating discussions and useful comments. We would like to thank two anonymous referees for their constructive comments on the manuscript 


\section{Appendix}

Computations of fluxes of dioxygen $\left(\dot{J}_{O}\right)$, carbon dioxide $\left(\dot{J}_{C}\right)$, water $\left(\dot{J}_{H}\right)$ and ammonia $\left(\dot{J}_{N}\right)$ comes with the specification of the stoichiometry of elements ( matrix $n_{\mathcal{M}}$ of chemical indices) and the ratios of $\mathrm{C}, \mathrm{H}, \mathrm{O}, \mathrm{N}$ to $\mathrm{C}$ in the generalized molecule which makes up the four organic compartments: $X$ (food), $V$ (structure), $E$ (reserve), $P$ (faeces) which are collected in matrix $n_{\mathcal{O}}$.

Chemical indices $n_{\mathcal{O}}$ and $n_{\mathcal{M}}$ (see Lika et al., 2011, this special issue) are as follows:

$$
n_{\mathcal{O}}=\left(\begin{array}{llll}
1.00 & 1.00 & 1.00 & 1.00 \\
1.80 & 1.80 & 1.80 & 1.80 \\
0.50 & 0.50 & 0.50 & 0.50 \\
0.10 & 0.10 & 0.10 & 0.10
\end{array}\right) \quad n_{\mathcal{M}}=\left(\begin{array}{llll}
1 & 0 & 0 & 0 \\
0 & 2 & 0 & 3 \\
2 & 1 & 2 & 0 \\
0 & 0 & 0 & 1
\end{array}\right)
$$

Organic fluxes are a weighted sum of the three basic powers in DEB theory: assimilation $\dot{p}_{A}$, dissipation $\dot{p}_{D}$ and growth $\dot{p}_{G}$. Assimilation and growth powers are defined in the main text (section 2.1). Somatic maintenance, maturity maintenance and maturation all contribute to the dissipation power such that $\dot{p}_{D}=\left[\dot{p}_{M}\right] L^{3}+(1-\kappa) \dot{p}_{C}$. Mineral fluxes are a weighted sum of the organic fluxes. Faeces production is linked only to assimilation so is zero for embryos.

The four organic fluxes, $\dot{J}_{X}$ flux of food (C-mol $X$ day $\left.{ }^{-1}\right), \dot{J}_{V}$ structure flux (C-mol $V$ day $\left.^{-1}\right), \dot{J}_{E}$ reserve flux $\left(\mathrm{C}-\mathrm{mol} E\right.$ day $\left.^{-1}\right)$, and $\dot{J}_{P}$ feces flux $\left(\mathrm{C}-\mathrm{mol} P\right.$ day $\left.^{-1}\right)$ are specified as follows :

$$
\begin{aligned}
& \dot{J}_{X}=-\frac{y_{X E}}{\bar{\mu}_{E}} f\left\{\dot{p}_{A m}\right\} L^{2} \\
& \dot{J}_{V}=\frac{\left[M_{V}\right]}{\left[E_{G}\right]}\left(\kappa \dot{p}_{C}-\left[\dot{p}_{M}\right] L^{3}\right) \\
& \dot{J}_{E}=\frac{1}{\bar{\mu}_{E}}\left(f\left\{\dot{p}_{A m}\right\} L^{2}-\dot{p}_{C}\right) \\
& \dot{J}_{P}=-y_{P X} \dot{J}_{X}
\end{aligned}
$$

and $f=0$ for embryos. The four mineral fluxes $\dot{J}_{C}\left(\operatorname{mol~C}\right.$ day $\left.^{-1}\right), \dot{J}_{H}\left(\mathrm{~mol} \mathrm{H}_{\mathrm{day}}{ }^{-1}\right), \dot{J}_{O}(\mathrm{~mol}$ $\left.\mathrm{O} \mathrm{day}^{-1}\right)$, and $\dot{J}_{N}\left(\mathrm{~mol} \mathrm{~N}\right.$ day $^{-1}$ ) are a weighted sum of organic fluxes (see Eqn.4.37 Kooijman, 2010, pp.139):

$$
\dot{J}_{\mathcal{M}}=-n_{\mathcal{M}}^{-1} n_{\mathcal{O}} \dot{J}_{\mathcal{O}}
$$

with:

$$
\dot{J}_{\mathcal{M}}=\left(\begin{array}{c}
\dot{J}_{C} \\
\dot{J}_{H} \\
\dot{J}_{O} \\
\dot{J}_{N}
\end{array}\right) \quad \text { and } \quad \dot{J}_{\mathcal{O}}=\left(\begin{array}{c}
\dot{J}_{X} \\
\dot{J}_{V} \\
\dot{J}_{E} \\
\dot{J}_{P}
\end{array}\right)
$$




\section{References}

Augustine, S., Gagnaire, B., Adam-Guillermin, and Kooijman, S. A. L. M. (2011). Developmental energetics of zebrafish, Danio rerio. Comp Biochem Physiol A, 159:275-283.

Bagatto, B., Pelster, B., and Burggren, W. W. (2001). Growth and metabolism of larval zebrafish: effects of swim training. J Exp Biol, 204:4335-4343.

Bailey, K. M. and Houde, E. D. (1989). Predation on the eggs of larvae of marine fishes and the recruitment problem. Adv Mar Biol, 25:1-83.

Best, J., Adatto, I., Cockington, J., James, A., and Lawrence, C. (2010). A novel method for rearing first-feeding larval zebrafish: polyculture with Type L saltwater rotifers (Brachionus plicatilis). Zebrafish, 7(3):89-295.

Blaxter, J. and Fuiman, L. (1990). The role of sensory systems of herring larvae in evading predatory fishes. $J$ mar biol Ass UK, 70:413-427.

Craig, J. F. and Fletcher, J. M. (1984). Growth and mortality of zebra fish, Brachydanio rerio (Hamilton Buchanan), maintained at two temperatures and on two diets. J Fish Biol, 25:43-55.

Cushing, D. (1975). Marine ecology and fisheries. Cambridge.

Eaton, R. C. and Farley, R. D. (1974). Growth and the reduction of depensation of zebrafish, Brachydanio rerio, reared in the laboratory. Copeia, 1:204-209.

Guillaume, J., Kaushik, S., and Bergot, P. ant Métailler, R. (1999). Nutrition et alimentation des poissons et crustacés. INRA.

Hjort, J. (1914). Fluctuations in the great fisheries of Northern Europe viewed in the light of biological research. Rapp P-V Reùn Cons Int Explor Mer, 20:1-228.

Houde, E. D. (1987). Early life dynamics and recruitment variability. Am Fish Soc Symp, 2:17-29.

Jardine, D. and Litvak, M. K. (2003). Direct yolk sac volume manipulation of zebrafish embryos and the relationship between offspring size and yolk sac volume. J Fish Biol, 63:388-397.

Kamler, E. (2005). Parent-egg-progeny relationships in teleost fishes: an energetics perspective. Rev Fish Biol Fisher, 15:399-421.

Kimmel, C. B., Ballard, W. W., Kimmel, S. R., Ullmann, B., and Schilling, T. F. (1995). Stages of embryonic development of the zebrafish. Dev Dynam, 203(3):253-310.

Kooijman, S. A. L. M. (2009a). Social interactions can affect feeding behaviour of fish in tanks. J Sea Res, 62:175-178.

Kooijman, S. A. L. M. (2009b). What the egg can tell us about its hen: Embryonic development on the basis of dynamic energy budgets. J Math Biol, 58(3):377-394.

Kooijman, S. A. L. M. (2010). Dynamic Energy Budget Theory for metabolic organization. Cambridge. 
Kooijman, S. A. L. M., Sousa, T., Pecquerie, L., Van der Meer, J., and Jager, T. (2008). From food-dependent statistics to metabolic parameters, a practical guide to the use of dynamic energy budget theory. Biol Rev, 83:533-552.

Leggett, W. C. and Deblois, E. (1994). Recruitment in marine fishes: is it regulated by starvation and predation in the egg and larval stages? Neth J Sea Res, 32(2):119-134.

Lika, K., Freitas, V., van der Veer, H. W., van der Meer, J., Wijsman, J. W. M., Pecquerie, L., Kearney, M. R., and Kooijman, S. A. L. M. (2011). Species diversity as reflected by the parameters of the standard DEB model: the covariation method of estimation. J Sea Res. To appear.

May, R. (1974). Larval mortality marine fishes and the critical period concept. In Blaxter, J., editor, The early life history of fish, pages 3-19. Springer-Verlag, Berlin.

Miller, T., Crowder, L., Rice, J., and Marschall, E. (1988). Larval size and recruitment mechanisms in fishes: toward a conceptual framework. Can J Fish Aquat Sci, 45:1657-1670.

Paradis, A. R., Pepin, P., and Brown, J. A. (1996). Vulnerability of fish eggs and larvae to predation: review of the influence of the relative size of prey and predator. Can J Fish Aquat Sci, 53:1226-1235.

Riveiro, I., Guisande, C., Lloves, M., Maneiro, I., and Cabanas, J. M. (2000). Importance of parental effects on larval survival in Sardina pilchardus. Mar Ecol Prog Ser, 205:249-258.

Rougier, F., Menudier, A., Bosgiraud, C., and Nicolas, J. A. (1996). Copper and zinc exposure to zebrafish, Brachydanio rerio (hamilton-buchaman): effects in experimental listeria infection. Ecotoxicol Environ Saf, $34: 134-140$.

Shepherd, T., Costain, K., and Litvak, M. K. (2000). The effect of development rate on swimming behaviour and escape response in American plaice (Hippoglosoides platessoides) larvae. Mar Biol, 137:737-745.

Sousa, T., Domingos, T., and Kooijman, S. A. L. (2008). From empirical patterns to theory: A formal metabolic theory of life. Philos Trans R Soc B, 363:2453-2464.

Sousa, T., Domingos, T., Poggiale, J. C., and Koijman, S. A. L. (2010). Dynamic energy budget restores coherence in biology. Philos Trans $R$ Soc B, 365:3413-3428.

Yin, M. C. and Blaxter, J. H. S. (1986). Morphological changes during growth and starvation of larval cod (Gadus morhua L.) and flounder (Platichthys flesus L.). J Exp Mar Biol Ecol, 104:215-228.

Zhang, R., Yang, J., and Xu, X. (2009). Depletion of zebrafish Tcap leads to muscular dystrophy via disrupting sarcomere-membrane interaction, not sarcomere assembly. Hum Mol Genet, 18(21):4130-4140.

Information about the DEB research program and its results can be found at http://www.bio.vu.nl/thb/deb/.

The Add_My_Pet collection can be found at http://www.bio.vu.nl/thb/deb/deblab/add_my_pet/. 